\title{
OPEN The therapeutic effects of adipose-derived mesenchymal stem cells on obesity and its associated diseases in diet-induced obese mice
}

\author{
Hala jaber ${ }^{1}$, Khodr Issa ${ }^{2,3}$, Ali Eid ${ }^{3,4,5}$ \& Fatima A. Saleh ${ }^{6 \bowtie}$
}

Obesity is a global public health concern associated with increased risk of several comorbidities. Due to the limited effectiveness of current therapies, new treatment strategies are needed. Our aim was to examine the effect of adipose-derived mesenchymal stem cells (AD-MSCs) on obesity and its associated diseases in a diet-induced obese (DIO) animal model. C57BL6 mice were fed with either high fat diet (HFD) or CHOW diet for 15 weeks. Obese and lean mice were then subjected to two doses of AD-MSCs intraperitoneally. Mice body weight and composition; food intake; blood glucose levels; glycated hemoglobin (HbA1c), intraperitoneal glucose tolerance test and atherogenic index of plasma (AIP) were measured. Pro-inflammatory cytokines, tumor necrosis factor- $\alpha$ and interleukin-6, were also determined. AD-MSCs treatment reduced blood glucose levels, HbA1c and AIP as well as improved glucose tolerance in DIO mice. In addition, MSCs caused significant attenuation in the levels of inflammatory mediators in HFD-fed mice. Taken together, AD-MSCs were effective in treating obesity-associated diabetes in an animal model as well as protective against cardiovascular diseases as shown by AIP, which might be partly due to the attenuation of inflammatory mediators. Thus, AD-MSCs may offer a promising therapeutic potential in counteracting obesity-related diseases in patients.

Obesity is a global health problem, particularly in light of its association with increased risk of several comorbidities, all-cause mortality and impaired quality of life ${ }^{1}$. The prevalence of obesity is increasing worldwide in adolescents and adults of both genders and has doubled between 1980 and $2016^{2}$. It is also expected that by $2030,57.8 \%$ of the population will be obese ${ }^{3}$. Moreover, obesity is associated with a spectrum of obesity-related morbidities resulting from complications affecting different aspects of physiology. These morbidities include hyperlipidaemia, cardiovascular diseases, and type 2 Diabetes ${ }^{4}$. Currently, a wide variety of interventions has been proposed for obesity and its related disorders in clinical settings ${ }^{5,6}$. However, effective therapies to cure obesity and its comorbidities are still lacking. Thus, investigators are in an urgent need to look for potential treatments that show effectiveness. Stem cell-based therapies currently hold great promise to treat various diseases including cardiovascular diseases (CVD); neurodegenerative diseases, muscular degenerative disorders; hematopoietic and immune system disorders; liver injuries; diabetes; arthritis; cancers as well as other diseases in our body that could take benefit of stem cell therapy ${ }^{7,8}$.

Particularly, Mesenchymal stem cells (MSCs) are an attractive candidate for a wide range of therapeutic applications ${ }^{9}$. Their relative ease of isolation from many tissues such as the bone marrow, adipose tissue and umbilical cord coupled with their multilineage differentiation capacity, have made them potential tools to treat many disease ${ }^{10}$. In recent years, adipose-derived MSCs (AD-MSCs) are emerging due to their abundance, ease

\footnotetext{
${ }^{1}$ Department of Nutrition and Dietetics, Faculty of Health Sciences, Beirut Arab University, Beirut, Lebanon. ${ }^{2}$ Department of Molecular Diagnostics, Doctors' Center Laboratories, Beirut, Lebanon. ${ }^{3}$ Department of Pharmacology and Toxicology, Faculty of Medicine, American University of Beirut, Beirut, Lebanon. ${ }^{4}$ Department of Basic Medical Sciences, College of Medicine, OU Health, Qatar University, Doha, Qatar. ${ }^{5}$ Biomedical and Pharmaceutical Research Unit, QU Health, Qatar University, Doha, Qatar. ${ }^{6}$ Department of Medical Laboratory Technology, Faculty of Health Sciences, Beirut Arab University, Beirut 115020, Lebanon. ${ }^{\square}$ email: f.saleh@ bau.edu.lb
} 
a

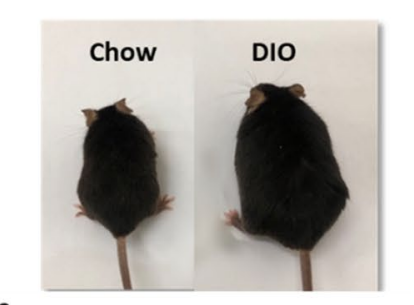

c

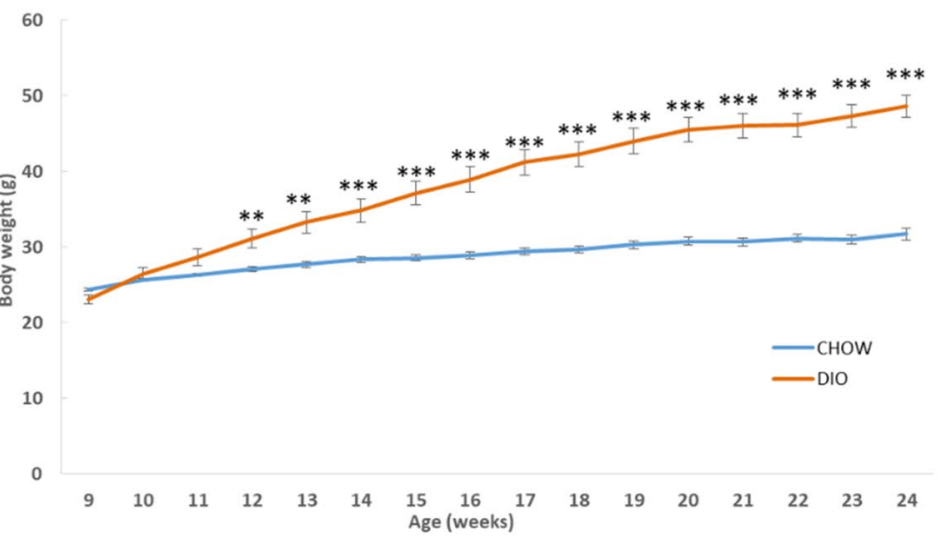

d

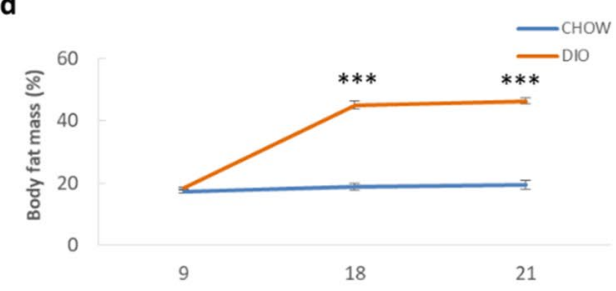

e

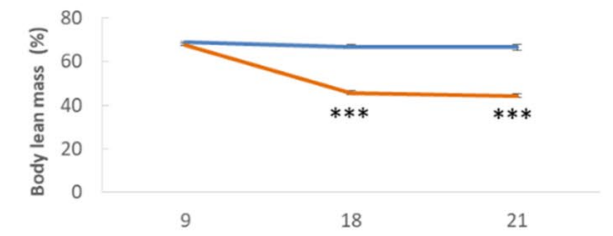

f

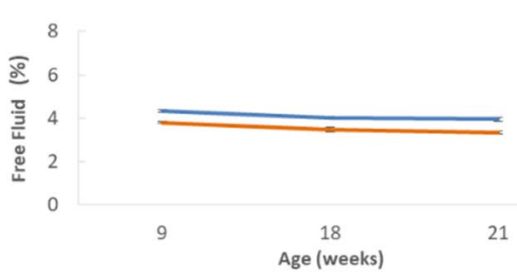

Figure 1. Generation of Diet-induced obese (DIO) mice by high-fat diet feeding for 15 weeks. (a) Representative images of DIO and CHOW mice (control). (b) Food intake of DIO and CHOW mice at 21 weeks of age. (c) Changes in body weights of DIO and CHOW mice undertaken weekly during the 15 week-feeding period. (d) Body-fat percentages, (e) body lean percentages and (f) free fluid percentages of DIO and CHOW mice at 9,18 and 21 weeks of age. $n=12,{ }^{* *} p<0.01,{ }^{* *} p<0.001$.

of collection, rapid expansion and high proliferation capacity ${ }^{11}$. Additionally, studies have confirmed that they can maintain the basic phenotype of MSCs over long-term culture up to Passage $10^{11}$. Stem cell therapy in obesity and its related diseases has been suggested by several animal studies but results seem to be unclear, making it far and difficult to move into clinical settings ${ }^{12-14}$. Therefore, well-designed studies are needed to determine efficacy in the animal model before proceeding to humans.

The purpose of this study was to investigate the effect of AD-MSCs on body weight, body composition, glycaemic control and dyslipidaemia in an in vivo animal model. An experimental model of obesity was established in mice under chronic high-fat diet (HFD) feeding for 15 weeks. The therapeutic potential of intraperitoneal $\mathrm{AD}-\mathrm{MSC}$ infusion to treat dietary-induced obesity and its associated disorders such as diabetes and CVD in a mouse model of HFD-induced obesity was elucidated in this study. The mechanism by which AD-MSCs produce their therapeutic effects was also explored.

\section{Results}

Generation of HFD-induced obese and diabetic mice. 8-week-old male C57BL/6 mice were allowed to adapt for 1 week to the new environment. Then, mice were fed for 15 weeks with either a high-fat diet (HFD) to generate diet-induced obese (DIO) mice or a standard CHOW diet to produce lean mice (Fig. 1a). Food intake was measured after 12 weeks of feeding. Nevertheless, there was no significant difference in food intake between DIO and CHOW mice (Fig. 1b). Regarding body weight, mice fed the HFD displayed significantly higher body weights than mice fed with the CHOW diet after only 3 weeks of feeding $(p<0.01)$. This increase in body weight in the HFD-fed mice continued throughout the 15 -week feeding period to reach $48.63 \pm 1.46 \mathrm{~g}$ in DIO mice as compared to $31.75 \pm 0.73 \mathrm{~g}(p<0.001)$ in CHOW mice at 24 weeks of age as shown in Fig. 1c. As presented in Fig. $1 \mathrm{~d}-\mathrm{f}$, there were alterations in body composition parameters upon high fat dieting, as determined using Minispec LF110 body composition analyzer. At baseline, no significant differences in parameters were observed. However, after 9 weeks of HFD feeding, there was significant elevation $(p<0.001)$ in fat mass percentage in the HFD group (Fig. 1d). At week 21, DIO mice had $46.4 \%$ body fat mass as compared to $19.5 \%$ in CHOW mice $(p<0.001)$. On the other hand, lean mass percentage decreased significantly in DIO mice at weeks 18 and 21 $(p<0.001)$ (Fig. 1e). However, free fluid content was not significantly different between both mice groups at all time points (Fig. 1f). It can be concluded that DIO mice continued to gain weight over the time period of the study and that was mainly due to the increase in total body fat mass rather than lean mass.

Blood glucose levels were also measured regularly every 2 weeks in both mice groups. After only 2 weeks on HFD, blood glucose significantly increased from $135.5 \pm 1.33 \mathrm{mg} / \mathrm{dl}$ to $152.5 \pm 2.25 \mathrm{mg} / \mathrm{dl}(p<0.01)$ and remained elevated to reach $187.7 \pm 5.6 \mathrm{mg} / \mathrm{dl}$ on week 21 as compared to $140.5 \pm 1.99 \mathrm{mg} / \mathrm{dl}$ in CHOW-fed mice $(p<0.001)$ (Fig. 2a). However, blood glucose measurements showed no significant increase in the control group throughout 


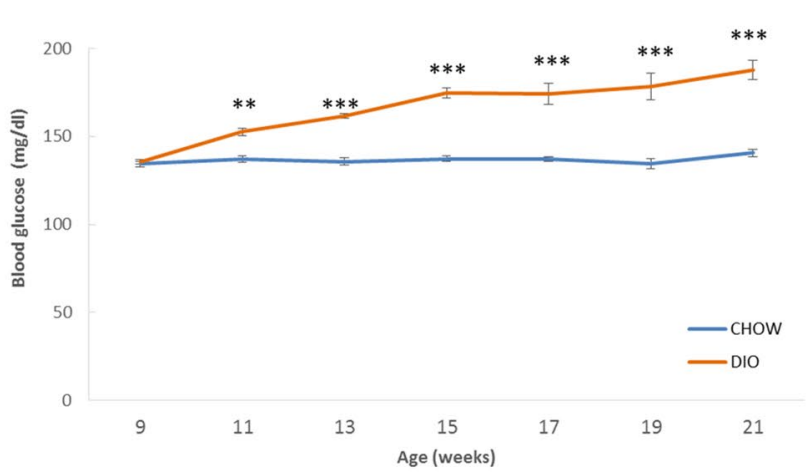

b

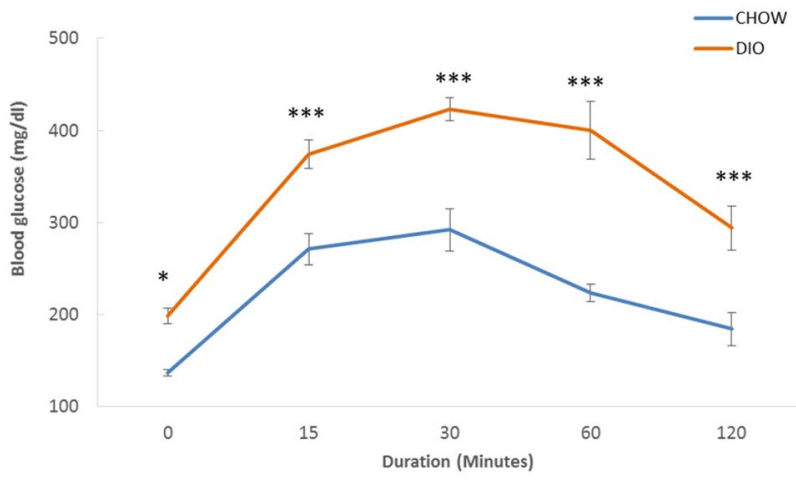

Figure 2. Blood glucose levels and Glucose tolerance in DIO and CHOW mice. (a) Changes in blood glucose levels of DIO and CHOW mice that were determined every 2 weeks during the 15 week-feeding period. (b) Intraperitoneal Glucose Tolerance Test (IPGTT) was performed at the end of the feeding period. $\mathrm{n}=12$, ${ }^{*} p<0.05,{ }^{* *} p<0.01,{ }^{* *} p<0.001$.

the 15 -week feeding period. This indicates that blood glucose levels were altered by the HFD thus inducing hyperglycemia. A two-hour intraperitoneal glucose tolerance test (IPGTT) was also performed after 15 weeks of diet feeding to assess glucose homeostasis in mice. After $6 \mathrm{~h}$ of fasting $(\mathrm{t}=0 \mathrm{~min})$, there was a significant increase $(\mathrm{P}<0.05)$ in blood glucose levels in DIO mice $(199 \pm 8.4 \mathrm{mg} / \mathrm{dl})$ versus control mice $(136.6 \pm 3.63 \mathrm{mg} /$ dl) (Fig. 2b). Now in order to determine the body's ability to metabolize glucose, a $2 \mathrm{~g} / \mathrm{kg}$ of glucose bolus was injected intraperitoneally (IP). DIO mice showed a rise in glucose levels (374 $\pm 15.5 \mathrm{mg} / \mathrm{dl})$ after $15 \mathrm{~min}$ of infusion with a peak occurring after $30 \mathrm{~min}(423 \pm 12.6 \mathrm{mg} / \mathrm{dl})(p<0.001)$ (Fig. $2 \mathrm{~b})$. Two hours later, both groups showed a reduction in glucose levels with the DIO group displaying significantly $(p<0.001)$ higher blood glucose level as compared with the CHOW-fed mice with a mean of $294 \pm 24 \mathrm{mg} / \mathrm{dl}$ and $184.3 \pm 18.14 \mathrm{mg} / \mathrm{dl}$, respectively. These data suggest that the HFD caused glucose intolerance in mice.

Effect of AD-MSCs on body weight and composition. We examined the impact of IP injection of AD-MSCs on obesity and related comorbidities. After chronic HFD feeding (15 weeks), six mice of DIO and CHOW groups were injected with $4.2 \times 10^{7} \mathrm{AD}-\mathrm{MSCs} / \mathrm{kg}$, while the control groups were injected with DMEM/ F12 medium. A second dose followed after 10 weeks. After 15 weeks of feeding at baseline (day 0 ), the body weights of both DIO groups, HFD + MEDIA and HFD + ADMSCs, were significantly high $(p<0.001)$ and reached around $49.35 \pm 1.42 \mathrm{~g}$ and $49.28 \pm 2.3 \mathrm{~g}$, respectively, as compared with the control groups, ND + media and $\mathrm{ND}+\mathrm{ADMSCs}$. However, at day 112, the body weights of both DIO groups (HFD + MEDIA and HFD + ADMSCs) decreased and reached $37.75 \pm 2.6 \mathrm{~g}$ and $37.36 \pm 2.47 \mathrm{~g}$, but there were neither significant differences between HFD + MEDIA and ND + MEDIA groups, nor between HFD + ADMSCs and ND + ADMSCs groups (Fig. 3a).

Body composition analysis, a method used to measure the percentages of fat mass, lean mass and free fluids, was performed. DIO groups had significantly $(p<0.001)$ higher percentages of fat mass $(52 \%$ and $46.7 \%)$ after chronic HFD feeding (day 7) as compared to controls (23\%); however, after AD-MSCs treatments, the percentages of fat mass decreased significantly in HFD group from $46.73 \pm 4.02 \%$ to $32.27 \pm 2.59 \%$ in a trend similar to that of HFD + MEDIA group (Fig. 3b). On the other hand, the lean mass \% (Fig. 3c) and fluid content $\%$ (Fig. 3d) were significantly lower in HFD-fed mice compared to CHOW-fed controls at baseline. Fifteen weeks after the first AD-MSCs administration, no significant changes were observed in both parameters in HFD-fed mice compared to controls.

Effect of AD-MSCs on blood glucose levels, HbA1c and GTT. At baseline (day 0), HFD + MEDIA and HFD + ADMSCS groups showed significantly $(p<0.001)$ elevated blood glucose levels reaching $185.25 \pm 10.4 \mathrm{mg} /$ $\mathrm{dl}$ and $181.8 \pm 6.15 \mathrm{mg} / \mathrm{dl}$, respectively (Fig. 4a,b). Blood glucose levels at the end of the 16-week experimental period significantly decreased to $135.4 \pm 1.63 \mathrm{mg} / \mathrm{dl}$ in the DIO mice treated with AD-MSCs as compared to those treated with media $(168.5 \pm 11.2 \mathrm{mg} / \mathrm{dl})$. Normal groups (ND + MEDIA and ND + ADMSCs) showed normal glucose levels (Fig. 4a,b). Furthermore, HbA1c levels performed 10 weeks after first intervention, measured $6 \pm 0.1 \%$ in HFD + MEDIA group as compared to $5.6 \pm 0.12 \%$ in HFD + ADMSCs, but this was not significant (Fig. 4c). However, HbA1c levels at 6 weeks after second injection were significantly decreased $(p<0.001)$ in HFD-fed mice treated with AD-MSCs reaching 5.6 $\pm 0.1 \%$ (Fig. $4 \mathrm{~d}$ ).

In addition to blood glucose and HbAlc tests, IPGTT was performed 8 weeks after first injection. HFD + MEDIA group compared to control groups showed a significant increase $(p<0.001)$ in glucose level $>340 \mathrm{mg} / \mathrm{dl}$ after $30 \mathrm{~min}$ of glucose injection which dropped to around $201.75 \pm 5.8 \mathrm{mg} / \mathrm{dl}$ after $2 \mathrm{~h}$. However, HFD + ADMSCs group showed a significant difference $(p<0.001)$ in glucose levels compared to control groups with the peak occurring at $30 \mathrm{~min}$ with a mean of $332.6 \pm 6.84 \mathrm{mg} / \mathrm{dl}$ and reaching $163 \pm 4.02 \mathrm{mg} / \mathrm{dl}$ at $2 \mathrm{~h}$ (Fig. 5a). 6 weeks after second injection, no significant differences in glucose levels among HFD + ADMSCs and control groups were observed (Fig. 5b). As a conclusion, AD-MSCs provided a positive effect on glycemic status and enhanced glucose disposal. 
a

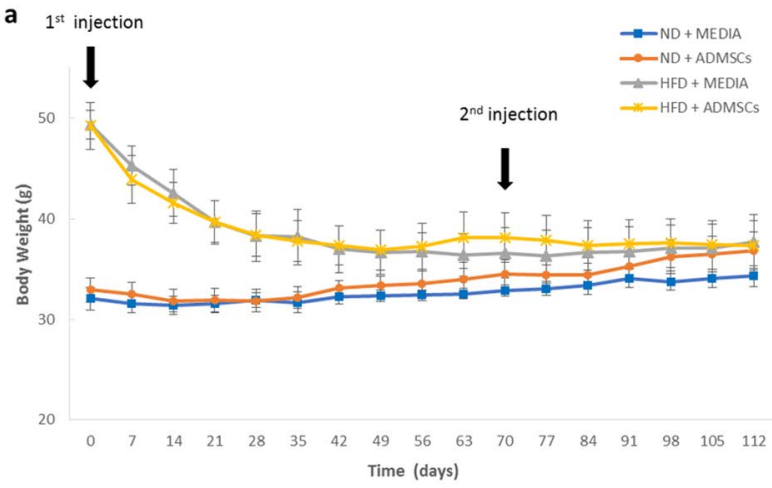

C

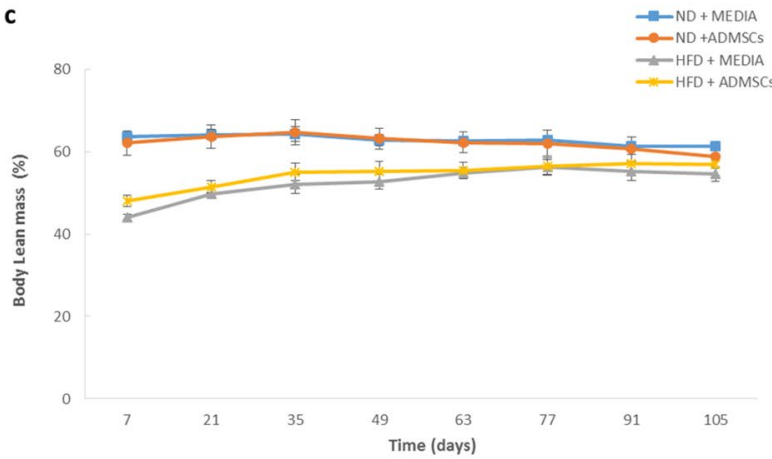

b

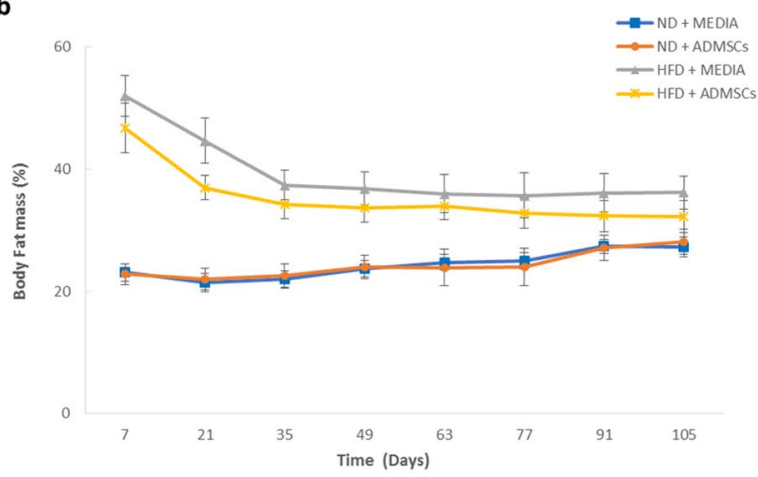

d

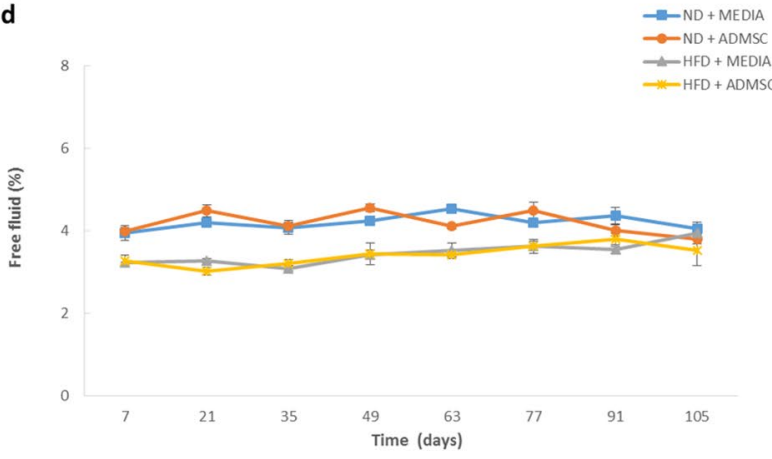

Figure 3. Changes in body weight and composition after MSC administration. (a) Changes in body weights (b) body fat mass percentages (c) body lean mass percentages and (d) free fluid percentages in normal mice and obese mice injected intraperitoneally (IP) with MEDIA and AD-MSCs at day 0 (1st injection) and day 70 (2nd injection). $\mathrm{n}=6$.

a

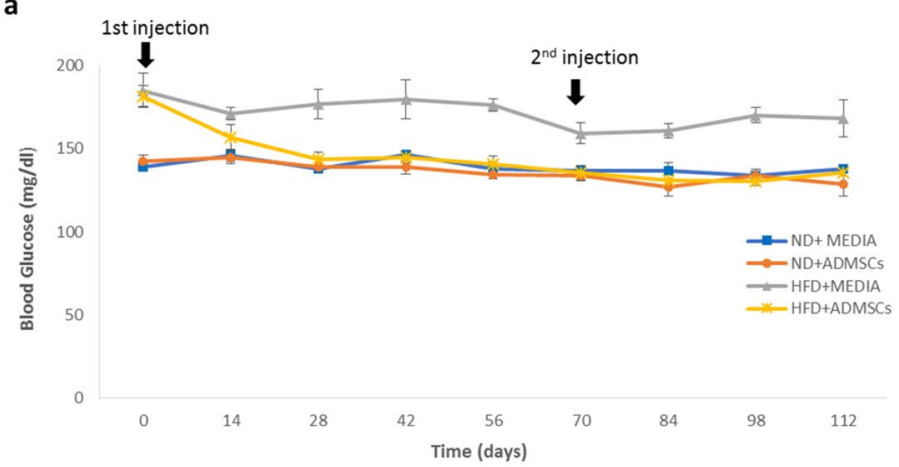

b

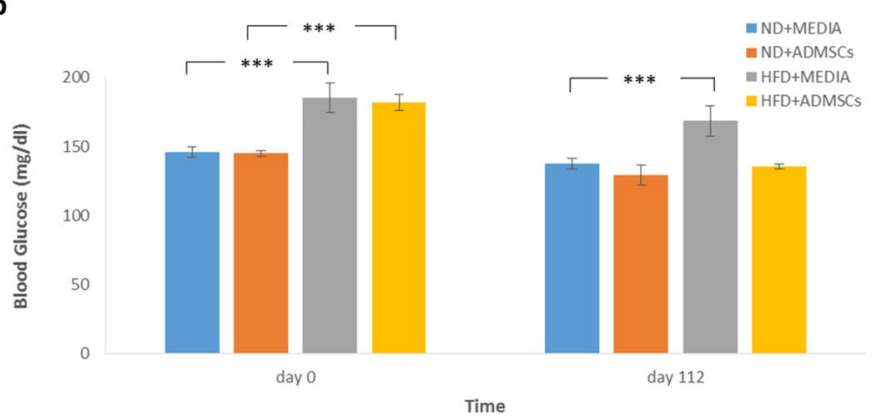

c

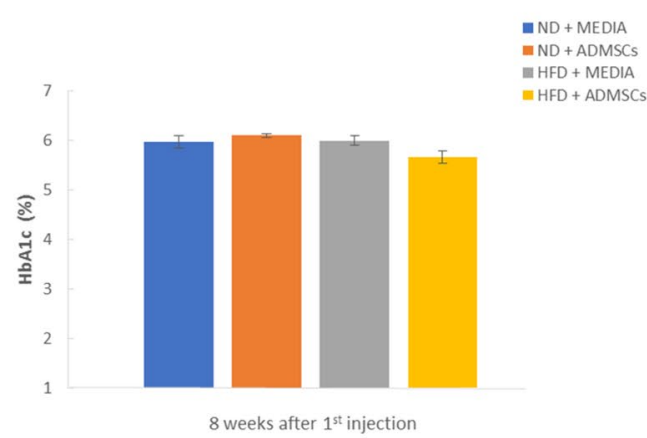

d

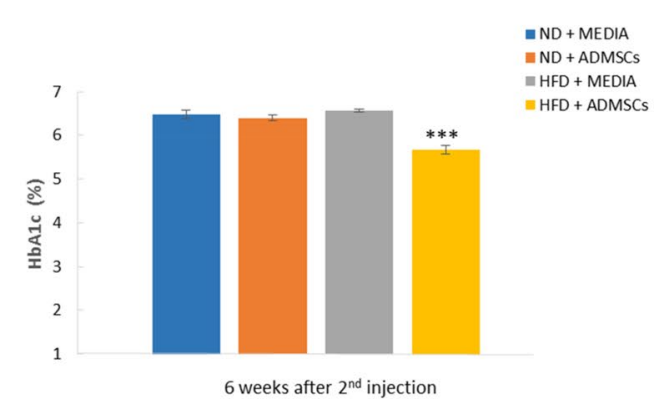

Figure 4. Blood glucose and glycated hemoglobin (HbAlc) levels after MSC administration. (a) and (b) Changes in blood glucose levels in ND- and HFD- fed mice injected IP twice with MEDIA and AD-MSCs, $\mathrm{n}=6$. (c) HbA1c levels at 8 weeks after 1st MSC injection and (d) 6 weeks after 2nd MSC injection in ND- and HFDfed mice. $n=6,{ }^{* * *} p<0.001$. 

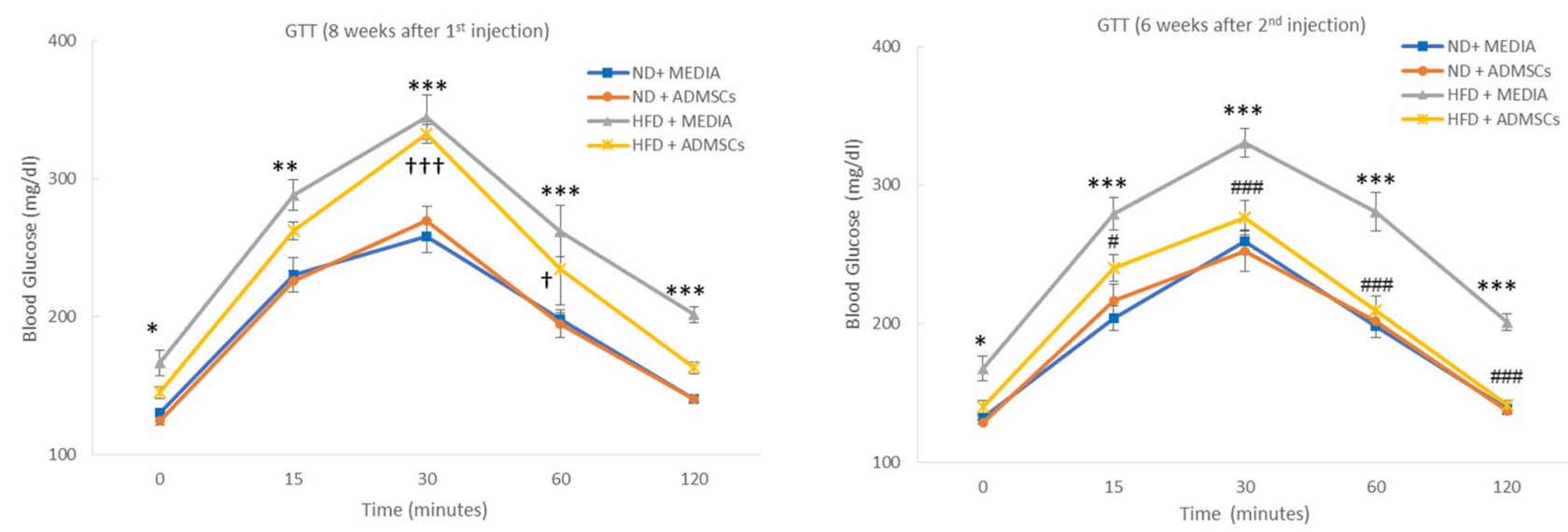

Figure 5. Effect of AD-MSC administration on Intraperitoneal Glucose Tolerance test (IPGTT). IPGTT was performed at (a) 8 weeks after 1st MSC injection and (b) 6 weeks after 2nd MSC injection in ND- and HFD- fed mice, $n=6$. ${ }^{\star}$ Indicates statistical difference between HFD + MEDIA group and ND groups; ${ }^{\dagger}$ Indicates statistical significance between HFD + ADMSC group and ND groups; ${ }^{*}$ Indicates statistical significance between HFD + ADMSC group and HFD + MEDIA group. ${ }^{\star} p<0.05,{ }^{* *} p<0.01,{ }^{* * *} p<0.001,{ }^{\dagger} p<0.05,{ }^{\dagger \dagger \dagger} p<0.001$, ${ }^{\#} p<0.05,{ }^{\# \#} p<0.001$.

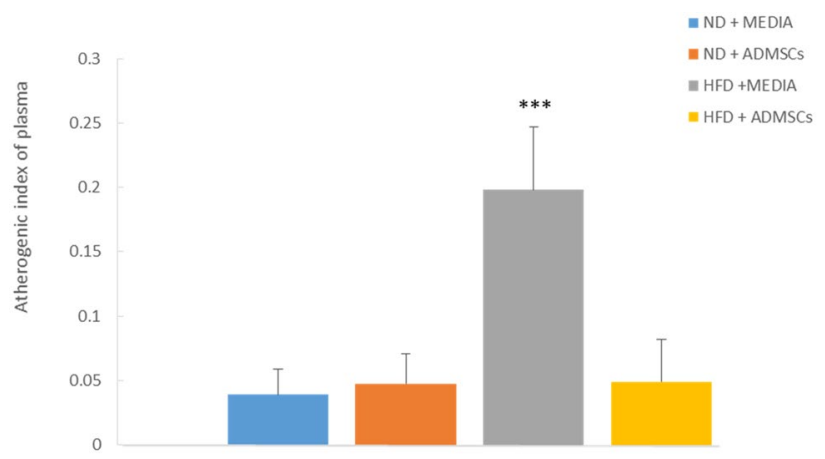

Figure 6. MSC treatment reduces the atherogenic index of plasma (AIP) in obese mice. AIP was measured at the end of the study in ND- and HFD- fed mice treated and untreated with AD-MSCs. $n=6,{ }^{* * *} p<0.001$.

Atherogenic index of plasma (AIP) levels. AIP is a very strong marker of cardiovascular diseases ${ }^{15}$. The effect of AD-MSCs on AIP was assessed by measuring HDL and TG values at the end of the study. Cardiovascular risk or AIP was the highest in HFD + MEDIA group as shown in Fig. 6. However, treatment of HFD group with AD-MSCs was sufficient to significantly reduce AIP to levels similar to those of normal diet groups.

Evaluation of TNF- $\alpha$ and IL- 6 levels. Pro-inflammatory cytokines, TNF- $\alpha$ and IL-6, were measured at the end of the experiment. High levels of TNF- $\alpha$ and IL- 6 were detected in both HFD and HFD + MEDIA groups; however, AD-MSCs transplantation caused a significant decrease in the protein levels (Fig. 7). Control groups (ND + MEDIA and ND + ADMSCs) showed normal levels of both cytokines.

\section{Discussion}

Obesity is a serious public health concern that increases the chances of developing numerous diseases such as diabetes, cardiovascular diseases and other comorbidities ${ }^{4}$. Throughout the past half century, scientific progression has allowed management of obesity and its associated diseases via several measures ${ }^{16}$. However, all these management options are not without limitations; thus, there is an urgent need for new interventions. Stem cell therapy seems to be promising as an alternative strategy to manage obesity and its related problems ${ }^{17,18}$. As a matter of fact, mesenchymal stem cells have become attractive candidates due to the vital role they play in adipogenesis and hence have been proposed as a novel therapeutic option ${ }^{19}$. The differentiation potential of MSCs, relative ease of isolation and expansion coupled with their immunomodulatory, anti-inflammatory and homing properties have made MSCs extensively studied both in vitro and in vivo for the treatment of many diseases ${ }^{17}$. The study presented here was undertaken to determine the effect of AD-MSCs intervention on body weight, body 
a

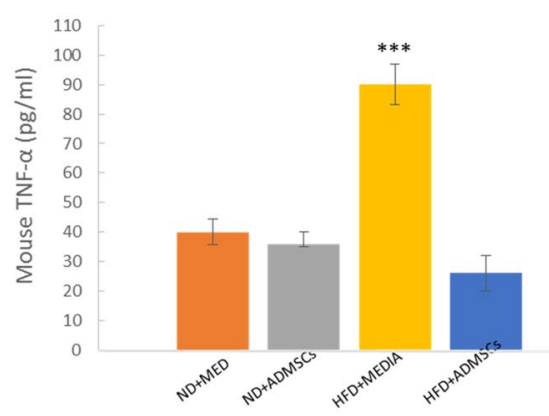

b

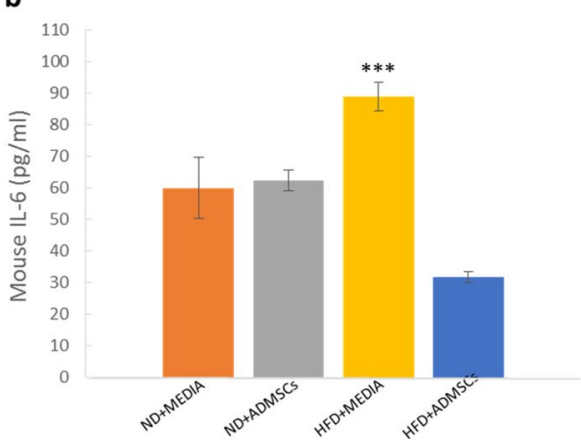

Figure 7. MSC treatment reduces pro-inflammatory cytokine levels in obese mice. (a) TNF- $\alpha$ and (b) IL-6 levels were measured by ELISA at the end of the study in ND- and HFD- fed mice treated and untreated with AD-MSCs. $n=6,{ }^{* * *} p<0.001$.

composition, hyperglycemia, glucose tolerance and cardiovascular risk in high fat diet-induced obese mouse model as well as its mechanism of action.

In our work, after 15-week chronic feeding period, DIO mice demonstrated substantial increase in body weight with a weight gain of $110 \%$. This was associated with significantly elevated fat mass ( $27.9 \%$ increase) and allevaited lean mass and free fluids. In contrast, mice fed with the CHOW diet remained healthy throughout the study. Herein, it was essential to inquire whether weight gained on a HFD was related to an increase in energy intake as a result of hyperphagia. Therefore, we measured the food intake in experimental mice over $24 \mathrm{~h}$. No significant change in food intake was observed in DIO and control groups. Nevertheless, other studies like the one presented by Licholai et al. concluded that HFD induces an overconsumption ${ }^{20}$. In parallel, DIO mice gradually developed hyperglycemia with blood glucose levels exceeding $187 \mathrm{mg} / \mathrm{dl}$ and impaired glucose tolerance at the end of experimental feeding; whereas, control mice remained lean and normoglycemic with no metabolic abnormalities. This is consistent with previous studies ${ }^{21-23}$.

To test our hypothesis, we examined the impact of IP injection of human AD-MSCs on body weight and composition in both DIO mice and their controls. One of the strengths of our study is the utilization of human cells rather than murine MSCs in animal disease model, as this will more closely mimic the human milieu and help accelerate the translation of stem cell therapy to clinical practice. A previous review demonstrated the effectiveness of human MSCs administered across several different cross-species models in 88 (93.6\%) out of 94 experimental studies ${ }^{24}$.

The body weight of both DIO groups (HFD + ADMSC and HFD + MEDIA) decreased significantly with time to reach weight similar to the normal diet groups after 16 weeks (112 days) of treatment. Despite no change in overall body weight at the end of the treatment period, stem cell therapy was sufficient to reduce body fat mass in DIO mice with slight increase in lean mass and free fluids. This was similar to other studies showing that administration of MSCs did not affect body weight in DIO animals ${ }^{25}$. However, it contradicts with others demonstrating that AD-MSCs can induce a decrease in body weight in HFD-fed mice ${ }^{26,27}$. Herein, we have also shown that AD-MSCs can ameliorate hyperglycemia induced by the HFD and improve glucose tolerance and glucosylated $\mathrm{Hb}$. In 2019, a study conducted by Shree et al. concluded that in obese/diabetic mice, AD-MSCs effectively decreased blood glucose levels and improved its tolerance in the experimental subjects ${ }^{28}$. It is well known that obesity is associated with increased risk of developing CVD. Therefore, to determine the effect of AD-MSC implantation on CVD risk in DIO mice, we measured AIP. AIP is a valuable novel marker to predict the risk of developing dyslipidemia and associated diseases such as cardiovascular diseases ${ }^{15}$. Reduced levels of AIP in HFD group treated with AD-MSCs showed the beneficial aspect and the possible anti-atherosclerotic effects of AD-MSCs. Our study agreed with a number of previous studies showing an improvement in serum lipid profile after AD-MSCs transplantation ${ }^{2,14,28}$. Moreover, when measuring inflammatory cytokines, it was striking that AD-MSCs treatment decreased TNF- $\alpha$ and IL-6 serum levels to concentrations similar to that of ND groups. This could explain the mechanism by which MSCs exert their hypoglycemic and cardioprotective effects by suppressing inflammatory markers, TNF- $\alpha$ and IL-6, that both play a role in the progression of diabetes and atherosclerosis ${ }^{29,30}$. Similar to our work, other laboratories have shown attenuation in inflammatory markers upon administration of MSCs in HFD-fed animal models ${ }^{31,32}$.

It is critically important to understand that obesity is associated with macrophage accumulation in adipose tissue $\mathrm{e}^{33,34}$. It has been reported that diet-induced obesity activates adipose tissue macrophages (ATM) into pro-inflammatory M1 macrophages that produce a variety of pro-inflammatory cytokines such as TNF- $\alpha$ and IL-6, which contribute to the development of diabetes and atherosclerosis ${ }^{35,36}$. In our study, in line with previous studies, MSC administration lowered the expression of pro-inflammatory cytokines ${ }^{37,38}$. Owing to their immunomodulatory and anti-inflammatory properties, we postulate that MSC infusion produced significant anti-diabetic effects via soluble factors in part through directing ATM into anti-inflammatory M2 state and subsequently suppressing the secretion of pro-inflammatory mediators, thus ameliorating the inflammatory microenvironment ${ }^{32,39}$.

In conclusion, our work showed effectiveness in treating obesity-associated diabetes as well as protective effect against CVD as shown by the AIP, which might be partly due to the attenuation of inflammatory mediators, 


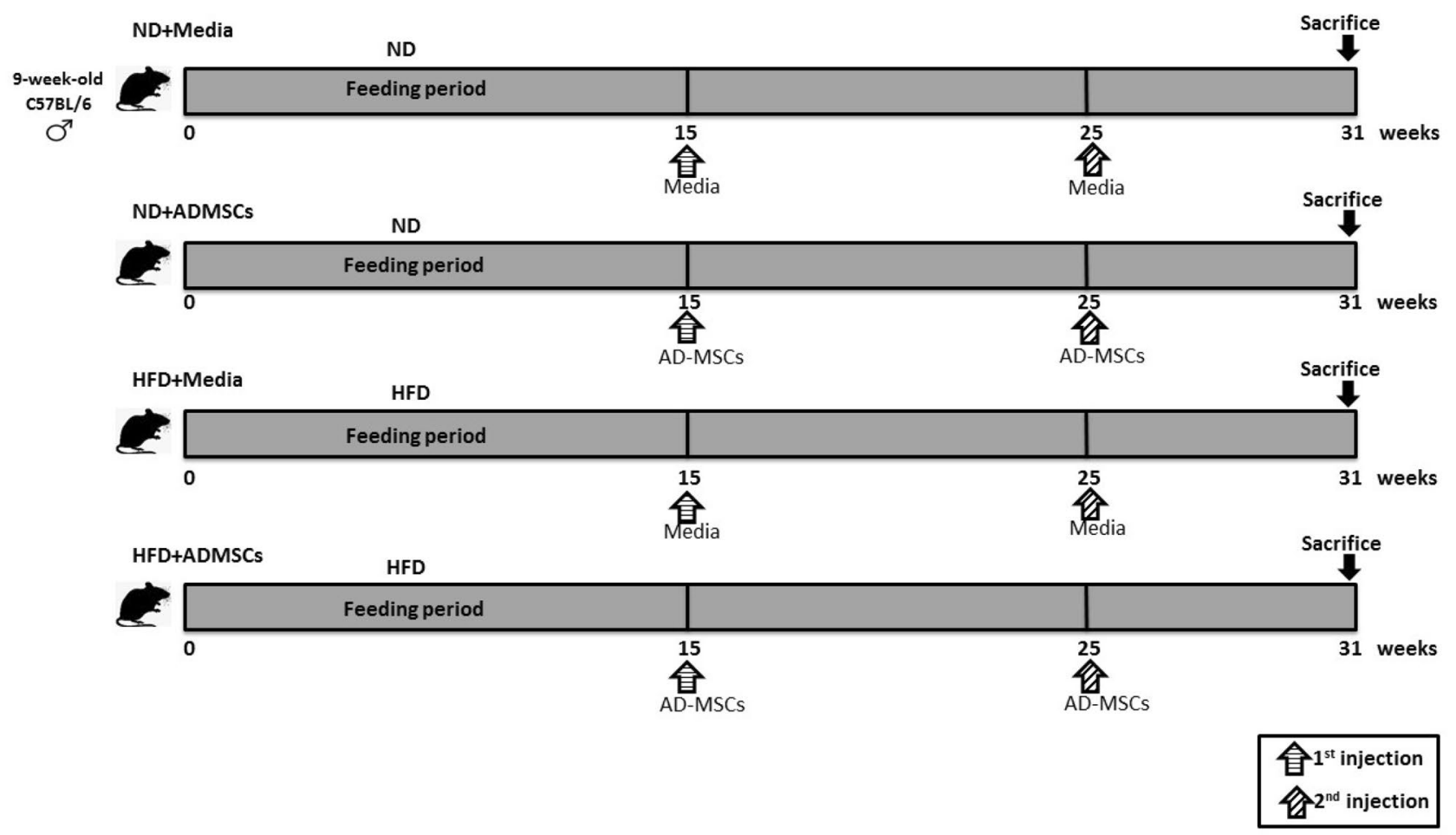

Figure 8. Schematic diagram of the experimental design of the study. Male C57BL/6 mice $(n=24)$ were fed with normal diet (ND) or (HFD) for 15 weeks. Following the feeding period, ND- and HFD-fed mice were returned to standard diet, then randomly divided into 2 groups each $(n=6)$. One group received 2 doses of adipose-derived mesenchymal stem cells (AD-MSCs); while the other received the media to serve as control.

TNF- $\alpha$ and IL-6. Despite the importance of our work in proving the functionality of using AD-MSCs for treating obesity-related diseases in an animal model, more future experiments should be conducted before moving forward into assessing AD-MSCs transplantation in treating obesity-concomitant diseases in humans.

\begin{abstract}
Methods
Animals and diets. Twenty-four male C57BL/6 mice aged 8 weeks (25-28 g) were hosted in a controlled environment at the Animal Care Facility at the American University of Beirut with free access to water and food and a 12-h light/dark cycle with a temperature of $22 \pm 2{ }^{\circ} \mathrm{C}$. A period of seven days was used as an adaptation period before conduction of the experimentation. All experimental procedures were performed in agreement with the NIH Guidelines for the Use of Animals in Research and approved (18-12-510) by the Institutional Animal Care and Use Committee (IACUC) at the American University of Beirut. Our study was also carried out in accordance with the ARRIVE (Animal Research: Reporting of In Vivo Experiments) guidelines ${ }^{40}$. Mice were then randomly divided into two groups. The normal diet (ND) group was fed a normal CHOW diet with $10 \%$ of total calories from fat and the other group fed a HFD (Research Diets, Inc, D12492, New Jersey, USA) with 60\% of calories from fat for a period of 15 weeks to generate the high fat diet-induced obese (DIO) mouse model.
\end{abstract}

Adipose-derived mesenchymal stem cells (AD-MSCs) culture. Human AD-MSCs (a kind gift from Reviva Regenerative Medicine Center, Bsalim, Lebanon) were maintained in a DMEM/F12 medium with 10\% FBS and $1 \%$ antibiotic-antimycotic at $37^{\circ} \mathrm{C}$ and $5 \% \mathrm{CO}_{2}$ atmosphere. The medium was changed every $48 \mathrm{~h}$ and cells were split when they reached $80-90 \%$ confluence. At passage 4 , cells were trypsinized and resuspended in DMEM/F12 media ready for animal treatment.

Experimental design. After 15 weeks of chronic feeding with the HFD or CHOW diet, the HFD was removed and half of the animals in each group $(n=6)$ were injected intraperitoneally (IP) with $4.2 \times 10^{7}$ cells/ $\mathrm{kg}$ AD-MSCs suspended in media (ND + ADMSCs and HFD + ADMSCs groups) and the other halves $(n=6)$ were injected with DMEM/F12 media as controls and labelled ND + MEDIA or HFD + MEDIA. A second treatment $\left(4.2 \times 10^{7}\right.$ cells $\left./ \mathrm{kg}\right)$ was repeated 10 weeks after the first injection. Similarly, control groups were injected with an equal volume of DMEM/F12 medium. The experimental procedure design is summarized in Fig. 8. All animals were sacrificed 6 weeks after the second injection and serum collected. For serum collection, blood was withdrawn from the retro-orbital sinus, centrifuged at $12 \mathrm{~g}$ for $15 \mathrm{~min}$ at $4{ }^{\circ} \mathrm{C}$, then serum stored at $-20^{\circ} \mathrm{C}$ for further analysis. 
Monitoring food intake, body weight and body composition. Mouse food intake was monitored before the first treatment injection at week 12 during a 24-h period. Body weights were also measured once per week and body composition analysis was performed using the Minispec LF110 Live Mice Analyzer (Bruker, USA) every 2 weeks for all mice. Briefly, the animal without anaesthesia was guided into a red restrainer to reduce the animal's anxiety. A tight-fitting plunger was also inserted into the restariner to maintain the mouse immobile during the duration of the scan, which is around $2 \mathrm{~min}$. The fat mass, lean mass, and free body fluid were measured before releasing the animal from the restrainer.

Measuring blood glucose, glycated hemoglobin and glucose tolerance. Blood glucose levels were measured every 2 weeks from a drop of blood by tail incision using Accu-Chek blood glucose meter and Accu-Chek Active glucose strips (ROCHE, Switzerland). Whereas, glycated hemoglobin (HbAlc) was assessed using the BIOHRMES HbAlc Analyzer (BIOHERMES, USA) at the 8th week following the first injection, and the 6th week following the second injection. Besides, the intraperitoneal glucose tolerance test (IPGTT) was performed using the Accu-Check blood glucose meter. Briefly, after six hours of fasting, mice body weights and fasting blood glucose levels were recorded $(\mathrm{t}=0 \mathrm{~min})$. Mice were then injected with $20 \%$ glucose. The volume of the glucose injection was calculated according to the formula: volume $=10 \times$ lean body mass $(\mathrm{g})^{41}$. Blood glucose levels were then measured at 15, 30, 60 and 120 min post-injection.

Determination of AIP levels. Triglycerides (TG) and high-density lipoprotein (HDL) cholesterol levels were measured at the end of the experiment using the Mission Cholesterol Monitoring System (ACON Labs Inc., USA) to determine the atherogenic index of plasma (AIP) that was calculated as AIP $=\log [\mathrm{TG} / \mathrm{HDL}]^{15}$.

Serum TNF- $\alpha$ and IL-6 protein levels. Tumor necrosis factor- $\alpha$ (TNF- $\alpha$ ) and interleukin-6 (IL-6) concentrations in the serum were assayed using the mouse TNF- $\alpha$ (ab208348) and IL-6 (ab222503) SimpleStep ELISA kits (Abcam) according to the manufacturer's instructions. The absorbance was measured spectrophotometrically using a Thermo Scientific Multiskan FC ELISA microplate reader at $450 \mathrm{~nm}$, and the cytokine concentrations were determined based on the optical densities obtained from the standards.

Statistical analysis. Data were analysed using the statistical package for social sciences (SPSS 24 for Windows) and presented as mean \pm SEM. A two-way analysis of variance (ANOVA) test was performed to assess the effects of the two variables (age, treatments) and their interactions on the body weight, blood glucose level and body composition (body fat mass, body lean mass, free fluid) of mice. This was followed by testing for statistically significant difference between variables using Bonferroni post-hoc test. $P$ value $<0.05$ was considered to be statistically significant.

Received: 9 November 2020; Accepted: 8 March 2021

Published online: 18 March 2021

\section{References}

1. Brown, W. V., Fujioka, K., Wilson, P. W. F. \& Woodworth, K. A. Obesity: Why be Concerned? Am. J. Med. 122, I-CO4 (2009).

2. Afshin, A. et al. Health effects of overweight and obesity in 195 countries over 25 years. N. Engl. J. Med. 377, 13-27 (2017).

3. Kelly, T., Yang, W., Chen, C. S., Reynolds, K. \& He, J. Global burden of obesity in 2005 and projections to 2030. Int. J. Obes. 32, 1431-1437 (2008).

4. Fruh, S. M. Obesity: Risk factors, complications, and strategies for sustainable long-term weight management. J. Am. Assoc. Nurse Pract. 29, S3-S14 (2017).

5. Yumuk, V. et al. European guidelines for obesity management in adults Hermann Toplak g for the obesity management task force of the European Association for the Study of Obesity. Obes. Facts 8, 402-424 (2015).

6. Wadden, T. A., Webb, V. L., Moran, C. H. \& Bailer, B. A. Lifestyle modification for obesity: New developments in diet, physical activity, and behavior therapy. Circulation 125, 1157-1170 (2012).

7. Lunn, J. S., Sakowski, S. A., Hur, J. \& Feldman, E. L. Stem cell technology for neurodegenerative diseases. Ann. Neurol. 70, 353-361 (2011).

8. Trounson, A. \& McDonald, C. Stem cell therapies in clinical trials: Progress and challenges. Cell Stem Cell 17, 11-22 (2015).

9. Saleh, F. A. \& Genever, P. G. Turning round: Multipotent stromal cells, a three-dimensional revolution?. Cytotherapy 13, 903-912 (2011).

10. Caplan, A. I. \& Correa, D. The MSC: an injury drugstore. Cell Stem Cell 9, 11-15 (2011).

11. Kozlowska, U. et al. Similarities and differences between mesenchymal stem/progenitor cells derived from various human tissues. World J. Stem Cells 11, 347-374 (2019).

12. Chen, J. et al. Adoptive transfer of syngeneic bone marrow-derived cells in mice with obesity-induced diabetes: selenoorganic antioxidant ebselen restores stem cell competence. Am. J. Pathol. 174, 701-711 (2009).

13. Ji, A.T.-Q., Chang, Y.-C., Fu, Y.-J., Lee, O. K. \& Ho, J. H. Niche-dependent regulations of metabolic balance in high-fat diet-induced diabetic mice by mesenchymal stromal cells. Diabetes 64, 926-936 (2015).

14. Liu, G.-Y. et al. Adipose-derived mesenchymal stem cells ameliorate lipid metabolic disturbance in mice. Stem Cells Transl. Med. 5, 1162-1170 (2016).

15. Niroumand, S. et al. Atherogenic Index of Plasma (AIP): A marker of cardiovascular disease. Med. J. Islam. Repub. Iran 29, 627-635 (2015).

16. Ruban, A., Stoenchev, K., Ashrafian, H. \& Teare, J. Current treatments for obesity. Clin. Med. J. R. Coll. Physicians Lond. 19, 205-212 (2019).

17. Saleh, F., Itani, L., Calugi, S., Grave, R. D. \& El Ghoch, M. Adipose-Derived Mesenchymal Stem Cells in the Treatment of Obesity: A Systematic Review of Longitudinal Studies on Preclinical Evidence. Curr. Stem Cell Res. Ther. 13, (2018).

18. Payab, M. et al. Stem cell and obesity: Current state and future perspective. in Advances in Experimental Medicine and Biology vol. 1089 1-22 (Springer New York LLC, 2018). 
19. Matsushita, K. \& Dzau, V. J. Mesenchymal stem cells in obesity: Insights for translational applications. Lab. Invest. 97, 1158-1166 (2017).

20. Licholai, J. A. et al. Why do mice overeat high-fat diets? How high-fat diet alters the regulation of daily caloric intake in mice. Obesity 26, 1026-1033 (2018).

21. Winzell, M. S. \& Ahrén, B. The high-fat diet-fed mouse: A model for studying mechanisms and treatment of impaired glucose tolerance and type 2 diabetes. in Diabetes vol. 53 (Diabetes, 2004).

22. Sampey, B. P. et al. Cafeteria diet is a robust model of human metabolic syndrome with liver and adipose inflammation: Comparison to high-fat diet. Obesity 19, 1109-1117 (2011).

23. Wang, C. Y. \& Liao, J. K. A mouse model of diet-induced obesity and insulin resistance. Methods Mol. Biol. 821, 421-433 (2012).

24. Li, J., Ezzelarab, M. B. \& Cooper, D. K. C. Do mesenchymal stem cells function across species barriers? Relevance for xenotransplantation. Xenotransplantation 19, 273-285 (2012).

25. Wang, W. et al. Feature Article: Transplantation of neuregulin 4-overexpressing adipose-derived mesenchymal stem cells ameliorates insulin resistance by attenuating hepatic steatosis. Exp. Biol. Med. 244, 565-578 (2019).

26. Hsiao, C. H. et al. Local injection of mesenchymal stem cells protects testicular torsion-induced germ cell injury Rocky Tuan; Timothy O'Brien. Stem Cell Res. Ther. 6, (2015).

27. Lee, C. W., Hsiao, W. T. \& Lee, O. K. S. Mesenchymal stromal cell-based therapies reduce obesity and metabolic syndromes induced by a high-fat diet. Transl. Res. 182, 61-74.e8 (2017).

28. Shree, N., Venkategowda, S., Venkatranganna, M. V., Datta, I. \& Bhonde, R. R. Human adipose tissue mesenchymal stem cells as a novel treatment modality for correcting obesity induced metabolic dysregulation. Int. J. Obes. 43, 2107-2118 (2019).

29. Current therapeutic approaches. Tousoulis, Di., Oikonomou, E., Economou, E. K., Crea, F. \& Kaski, J. C. Inflammatory cytokines in atherosclerosis. Eur. Heart J. 37, 1723-1735 (2016).

30. Cho, N. H. et al. Estimated association between cytokines and the progression to diabetes: 10-year follow-up from a communitybased cohort. J. Clin. Endocrinol. Metab. 105, 381-389 (2020).

31. Zhao, H. et al. Exosomes from adipose-derived stem cells attenuate adipose inflammation and obesity through polarizing M2 macrophages and beiging in white adipose tissue. Diabetes 67, 235-247 (2018).

32. Xie, Z. et al. Human umbilical cord-derived mesenchymal stem cells elicit macrophages into an anti-inflammatory phenotype to alleviate insulin resistance in type 2 diabetic rats. Stem Cells 34, 627-639 (2016).

33. Weisberg, S. P. et al. Obesity is associated with macrophage accumulation in adipose tissue. J. Clin. Invest. 112, 1796-1808 (2003).

34. Zeyda, M. \& Stulnig, T. M. Adipose tissue macrophages. Immunol. Lett. 112, 61-67 (2007).

35. Santos, E. W. et al. Short-term high-fat diet affects macrophages inflammatory response, early signs of a long-term problem. Brazilian J. Pharm. Sci. 55, 17561 (2019).

36. Lumeng, C. N., Bodzin, J. L. \& Saltiel, A. R. Obesity induces a phenotypic switch in adipose tissue macrophage polarization. J. Clin. Invest. 117, 175-184 (2007).

37. Li, Y. et al. Early intervention with mesenchymal stem cells prevents nephropathy in diabetic rats by ameliorating the inflammatory microenvironment. Int. J. Mol. Med. 41, 2629-2639 (2018).

38. Cao, M. et al. Adipose-derived mesenchymal stem cells improve glucose homeostasis in high-fat diet-induced obese mice. Stem Cell Res. Ther. 6, 208 (2015).

39. Yin, Y. et al. Human umbilical cord-derived mesenchymal stem cells direct macrophage polarization to alleviate pancreatic islets dysfunction in type 2 diabetic mice article. Cell Death Dis. 9, 1-18 (2018).

40. Percie du Sert, N. et al. The ARRIVE guidelines 2.0: Updated guidelines for reporting animal research. PLOS Biol. 18, e3000410 (2020).

41. Mouse Metabolic Phenotyping Centers MMPC Protocols Intraperitoneal glucose tolerance test (IP GTT).

\section{Acknowledgements}

The authors thank Dr. Samer Kharroubi from American University of Beirut for statistical guidance and Dr. Charbel Khalil from Reviva Regenerative Medicine Center, Lebanon, for the kind gift of AD-MSCs.

\section{Author contributions}

F.S. was involved in planning, designing the experiments and main supervision of the work. F.S. also designed the figures and performed statistical analysis. H.J. performed the experiments and processed the experimental data. K.I. aided in the experimental work and animal handling. F.S and H.J. drafted the manuscript. A.E. provided co-supervision and feedback on the final version of the manuscript.

\section{Funding}

This study was funded by Beirut Arab University (BAU) and the Lebanese National Council for Scientific Research (CNRS-L).

\section{Competing interests}

The authors declare no competing interests.

\section{Additional information}

Correspondence and requests for materials should be addressed to F.A.S.

Reprints and permissions information is available at www.nature.com/reprints.

Publisher's note Springer Nature remains neutral with regard to jurisdictional claims in published maps and institutional affiliations. 
(c) (i) Open Access This article is licensed under a Creative Commons Attribution 4.0 International cc) License, which permits use, sharing, adaptation, distribution and reproduction in any medium or format, as long as you give appropriate credit to the original author(s) and the source, provide a link to the Creative Commons licence, and indicate if changes were made. The images or other third party material in this article are included in the article's Creative Commons licence, unless indicated otherwise in a credit line to the material. If material is not included in the article's Creative Commons licence and your intended use is not permitted by statutory regulation or exceeds the permitted use, you will need to obtain permission directly from the copyright holder. To view a copy of this licence, visit http://creativecommons.org/licenses/by/4.0/.

(C) The Author(s) 2021, corrected publication 2021 